\title{
Autopercepção de Felicidade e Fatores Associados em Adultos de uma Cidade do Sul do Brasil: Estudo de Base Populacional
}

\author{
Self-Perceived Happiness and Associated Factors in Adults \\ in a Southern Brazilian City: A Population-Based Study
}

\author{
Diogo Luis Scalco*, ${ }^{*}$, Cora Luiza Araújo ${ }^{b} \&$ João Luiz Bastos ${ }^{c}$ \\ ${ }^{a}$ Grupo Hospitalar Conceição, Porto Alegre, Brasil, ${ }^{b}$ Universidade Federal de Pelotas, Pelotas, Brasil \\ \& 'Universidade Federal de Santa Catarina, Florianópolis, Brasil
}

\begin{abstract}
Resumo
Este estudo avaliou os níveis de autopercepção de felicidade e fatores associados em uma cidade do sul do Brasil. Foi realizado um estudo transversal, de base populacional, com 2.942 indivíduos adultos $(>20$ anos). Felicidade foi definida como "o grau, segundo o qual uma pessoa avalia positivamente a qualidade global de sua vida como um todo, no presente"; aferida por meio de uma pergunta associada a uma escala de faces. A prevalência global de felicidade foi de 73,4\%. Observou-se associação positiva entre a ocorrência de felicidade, escolaridade e nível econômico, bem como relação negativa de felicidade com desemprego. Ser mais jovem, entre os homens, e considerar-se mais religiosa, entre as mulheres, também se associaram a maiores prevalências de felicidade. Estar separada/divorciada ou ser viúva esteve associado a menores prevalências de felicidade entre as mulheres. Os resultados sugerem que a população investigada apresenta uma prevalência de felicidade alta e que fatores distintos estiveram associados à ocorrência deste desfecho entre homens e mulheres.

Palavras-chave: Felicidade; Estudos Transversais; Fatores Socioeconômicos.

Abstract

This study investigated self-perceived happiness and associated factors in inhabitants from a Southern Brazilian city. A cross-sectional population-based study was carried out with 2,942 individuals aged 20 years old and over. Self-perceived happiness was defined as the degree to which a person positively evaluates the overall quality of his/her present life as a whole. It was estimated through a single question using a "smiley scale". The prevalence of self-perceived happiness was $73.4 \%$ and it was positively associated with educational attainment and economic level, although it was negatively associated with unemployment. Young men and religious women also showed higher prevalences of happiness. Among women, being separated/divorced or widowed was associated with a lower prevalence of happiness. Results showed that the studied population presents high prevalence of self-perceived happiness. Distinct factors are also associated with happiness, according to the participants' gender.

Keywords: Happiness; Cross-Sectional Studies; Socioeconomic Factors.
\end{abstract}

A idéia de que felicidade é central na experiência de vida do homem remonta à antigüidade. $\mathrm{Na}$ Grécia antiga, quatro séculos antes de Cristo, o filósofo Aristippus afirmava que "o objetivo central da vida de cada ser humano seria maximizar seus prazeres” (Nettle, 2005). Na mesma linha, no século XIX, filósofos utilitaristas reivindicavam que o "objetivo primordial das políticas sociais seria gerar mais felicidade para um maior número de pessoas" (Geraldo, s.d.).

No campo político, a discussão é mais atual. O governo do pequeno Reino de Butão recentemente anunciou

\footnotetext{
* Endereço para correspondência: Grupo Hospitalar Conceição, Serviço de Saúde Comunitária, Posto de Saúde Jardim Itu, Rua Biscaia, 39, Cristo Redentor, Porto Alegre, RS, Brasil, CEP 91380-030. E-mail: dscalco@gmail.com
}

que o objetivo a ser alcançado por suas políticas públicas seria o aumento da "felicidade nacional bruta" ao invés do "produto nacional bruto" (ou produto interno bruto [PIB]; Mancall, 2004). Em países, como a Inglaterra, a pretensão não parece ser tão grande, mas planejadores de políticas de saúde discutem a necessidade do uso de estimativas de satisfação de vida e felicidade da população como indicadores de bem-estar populacional (Seymour, 2007).

Crescente importância tem sido dado ao tema no Brasil e em outros países por meio de editoriais em revistas médicas de grande circulação (Delamothe, 1996), informações e reportagens na mídia escrita, falada e eletrônica (Cowley, 2002; Fresneda, 2007; Moreira, 2007). Tal fato pode ser ilustrado por meio de uma busca na base de dados PubMed com os descritores "happiness", "public health" e "epidemiology" nos períodos de 1980- 
1999 e 2000-2007. A produção científica localizada com "happiness" perfaz $14,1 \%(N=448)$ e $17,1 \%(N=$ $731)$ de todos os trabalhos encontrados com "public health" e "epidemiology" nos períodos destacados, respectivamente.

Paralelamente, passam a despontar estudos demonstrando associação entre felicidade e estados correlatos com desfechos positivos em saúde. Barak (2006) apresenta uma revisão de literatura demonstrando associação de afeto positivo com melhor resposta imune. De maior relevância foi um estudo longitudinal com freiras acompanhadas por décadas, o qual apontou que aquelas com mais afeto positivo, demonstrado por meio da análise de anotações em seus diários quando jovens, tiveram uma expectativa de vida cerca de dez anos maior do que aquelas classificadas com menos afeto positivo (Danner, Snowdon, \& Friesen, 2001). De modo semelhante, Koivumaa-Honkanen et al. (2000) concluíram que através de um questionário com perguntas sobre satisfação de vida foi possível predizer menores taxas de mortalidade numa população de finlandeses adultos, especialmente entre indivíduos do sexo masculino.

Estudar o tema felicidade, entretanto, é desafiador, a começar pela dificuldade em definí-la. Considerada por Diener (2000) como um termo coloquial para bem-estar subjetivo e sinônimo de qualidade de vida para dois terços dos indivíduos em um estudo com profissionais de saúde realizado na Inglaterra (McKevitt, Redfern, La-Placa, \& Wolfe, 2003), felicidade será no presente trabalho entendida de acordo com a definição de Veenhoven. Este autor a descreve como um construto semelhante à satisfação de vida: "o grau, segundo o qual uma pessoa avalia positivamente a qualidade global de sua vida como um todo, no presente"(Veenhoven, 1997). Em outras palavras, felicidade será entendida como o quanto a pessoa está satisfeita com, ou gosta da vida que leva. Atem-se à apreciação que o indivíduo faz de sua vida no presente, ainda que esta possa sofrer influências do afeto momentâneo, de eventos passados na vida ou de perspectivas futuras.

Estudos apontam que, embora construtos como felicidade e afeto positivo apresentem validade discriminatória entre si, representam medidas de bem-estar com alto grau de correlação (Lucas, Diener, \& Su, 1996; Sandvik, Diener, \& Seidlitz, 1993). Tal afirmação, somada ao relatado no próximo parágrafo, justificam a opção dos autores pela utilização de comparações dos achados desta pesquisa com os de outras, que utilizaram diferentes desfechos de bem-estar.

Estudos epidemiológicos que apresentem informações a respeito dos níveis de autopercepção de felicidade populacionais e de fatores associados são relativamente escassos no Brasil, ainda que no mundo venham crescendo nos últimos anos. Este estudo objetivou estudar a percepção de indivíduos adultos acerca de sua condição de felicidade e verificar a associação com algumas características demográficas, socioeconômicas e comportamentais.

\section{Métodos}

Entre outubro de 2007 e janeiro de 2008, realizou-se um estudo transversal de base populacional na zona urbana do município de Pelotas, Rio Grande do Sul, Brasil. Esta investigação foi conduzida por uma turma de mestrandos do Programa de Pós-Graduação em Epidemiologia da Universidade Federal de Pelotas. Os dados foram coletados através de um único questionário com questões gerais e itens específicos do interesse de cada pesquisador (Barros et al., 2008).

Pelotas está situada ao sul do estado do Rio Grande do Sul e, segundo estimativa do Instituto Brasileiro de Geografia e Estatística (IBGE, 2007), possuía 339.934 habitantes no ano de 2007 , sendo que $93,2 \%$ residiam na área urbana do município. A população-alvo do presente estudo incluiu os moradores da área urbana, de 20 anos ou mais de idade, excluindo-se os indivíduos institucionalizados, residindo em prisões ou instituições de longa permanência para idosos.

Para o cálculo do tamanho da amostra, foi utilizado o programa Epi-Info. Considerando prevalências de autopercepção de felicidade de $60 \%, 70 \%$ e $80 \%$, nível de confiança de $95 \%$, poder de $80 \%$ e erro aceitável de dois pontos percentuais, acréscimo de $10 \%$ para perdas e recusas e mais $50 \%$ para compensar o efeito de delineamento amostral, a amostra necessária deveria incluir 2.516 indivíduos. Num segundo momento, procedeu-se outro cálculo para verificar a associação entre autopercepção de felicidade e as variáveis independentes, considerando os seguintes parâmetros: nível de confiança de $95 \%$, poder de $80 \%$ e risco relativo a ser detectado de 1,2 , levando-se em conta as prevalências conhecidas ou estimadas das variáveis independentes a serem estudadas na população de Pelotas. Após acréscimo de $10 \%$ para perdas ou recusas e $50 \%$ para o efeito de delineamento amostral, estimou-se um tamanho amostral de 1.635 indivíduos. O número final de entrevistados ultrapassou estes valores, tendo em vista que uma amostra maior foi necessária para atender os objetivos de estudos de outros pesquisadores envolvidos no trabalho.

A amostra foi selecionada em dois estágios. As unidades primárias de amostragem foram os 404 setores censitários urbanos, residenciais, de Pelotas. Após estratificação pela renda mensal média do chefe da família (IBGE, 2000), foram sorteados 126 setores, com probabilidade proporcional ao tamanho. Uma vez que um setor foi sorteado duas vezes, o número final de setores da amostra totalizou 125. Em cada setor censitário foram amostrados de forma sistemática, aproximadamente, 11 domicílios, com exceção do setor que foi sorteado duas vezes, o qual teve o dobro de domicílios visitados.

Todos os domicílios foram previamente visitados pelos pesquisadores responsáveis, com o intuito de convidar os moradores a participarem do estudo, além de identificar a idade, o sexo e o número de moradores. A coleta de dados ocorreu por meio da aplicação de questionários 
padronizados por entrevistadoras treinadas que desconheciam os objetivos e as hipóteses do estudo. Os questionários foram testados e aprimorados a partir da realização de um estudo-piloto em um setor censitário não incluído na amostra.

Ao final do trabalho de campo, foram entrevistados 2.986 indivíduos entre os 3.180 elegíveis, o que corresponde a uma taxa de perdas/recusas de $6,1 \%$. Dentre os entrevistados, 44 (1,5\%) foram excluídos por não poderem responder por si mesmos ao questionário em decorrência de impedimento físico (incluídos aqueles com dificuldade visual importante) ou incapacidade mental relatada por co-habitantes do domicílio. Dessa forma, a amostra final incluída na presente análise foi composta por 2.946 indivíduos.

O desfecho investigado foi autopercepção de felicidade, mensurada através de uma pergunta acompanhada da escala de faces de Andrews e Whitey, apresentada na obra de McDowell e Newell (1996; Figura 1). Foram consideradas felizes as pessoas que apontaram as faces $\mathrm{A}$ ou $\mathrm{B}$.

Agora vou the mostrar algumas faces que expressam vários sentimentos, desde uma pessoa que se sente muito feliz [apontar a primeira face] até uma pessoa que se sente muito infeliz [apontar para a última face passando por todas a demais faces intermediárias].

Qual dessas faces mostra melhor o jeito como o (a) senhor (senhora) se sente, pensando em sua vida como um todo?

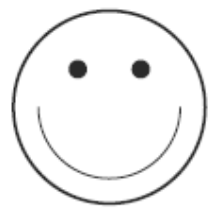

A

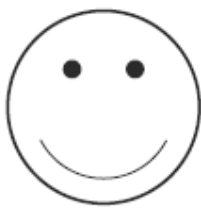

B

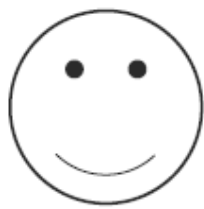

C

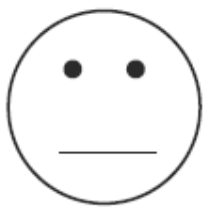

$\mathrm{D}$

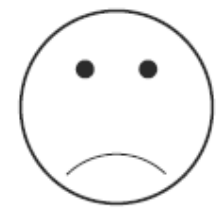

$\mathrm{E}$

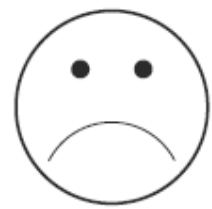

$\mathrm{F}$

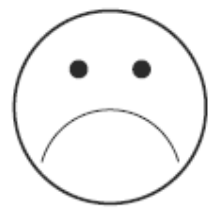

G

Figura 1. Instrumento utilizado para determinação de autopercepção de felicidade (McDowell \& Newell, 1996).

Cabe destacar que a escolha por este instrumento de único-item (uma única pergunta) foi precedida por uma rigorosa revisão da literatura e por discussão entre os autores deste manuscrito. Embora medidas de múltiplos itens tenham como vantagem uma maior capacidade de captar os diversos componentes do construto felicidade, além de reduzir erros de mensuração, não foram encontradas na literatura publicações de instrumentos de múltiplos itens que tivessem sido submetidos a um processo de adaptação transcultural para uso no contexto brasileiro. Assim, atendendo ao caráter preliminar do estudo, escolhemos a escala de faces como instrumento de um único item para avaliação do construto felicidade.

A literatura aponta que a confiabilidade teste-reteste deste item é de 0,7 (McDowell \& Newell, 1996), quando avaliada ao fim de uma mesma entrevista, e de 0,6 (Veenhoven, 1997), considerando-se um intervalo de sete dias entre a primeira e a segunda aplicações do item. Diener (1984) explicita que a validade e confiabilidade de medidas de único-item sugerem que sejam adequadas para grandes inquéritos populacionais, dada sua facilidade de aplicação.

Somado a estas considerações, ressalta-se que foi realizado um rigoroso pré-teste do instrumento em oitenta indivíduos não incluídos na amostra final, porém com perfil sócio-demográfico semelhante. A compreensão do item foi avaliada pela técnica de "think-aloud", na qual o participante expressa com suas próprias palavras o que o mesmo representa para ele. Este procedimento nos in- dicou adequada validade do item, embora estratégias mais rigorosas não tenham sido empregadas para avaliar este aspecto - a validade da mensuração - e suas diversas facetas.

A opção de dicotomizar o desfecho, com a escolha das duas faces mais "felizes", embora possa ser um fator que limite a comparação com outros estudos, foi uma opção dos autores, baseada na análise da literatura e outros estudos e antecedeu a realização da pesquisa.

As variáveis independentes utilizadas foram:

Demográficas - sexo: masculino ou feminino; idade: 20-29, 30-39, 40-49, 50-59 ou 60 anos ou mais; cor/raça autoclassificada como branca, preta/parda (agrupadas em uma única categoria por representarem, neste estudo, grupos populacionais semelhantes quanto à freqüência do desfecho) e outros. Esta última variável foi incluída no estudo com o objetivo de captar alguma possível desigualdade no desfecho que não fosse contemplada pelas demais variáveis; e, situação conjugal: casado/com companheiro, solteiro, separado ou viúvo.

Socioeconômicas - escolaridade: 0, 1-4, 5-8, 9-11 ou 12 anos ou mais de estudo; nível econômico: A, B, C, D/ E (Associação Brasileira de Empresas de Pesquisa [ABEP], 2005); situação de trabalho: trabalhando, desempregado, dona de casa, aposentado/pensionista (agrupados em uma única categoria por representarem grupos populacionais semelhantes entre si - média de idade e prevalência do desfecho semelhantes), outros; número de habitantes no domicílio: 1, 2, 3, 4 e 5 ou mais. 
Scalco, D. L., Araújo, C. L. \& Bastos, J. L. (2011). Autopercepção de Felicidade e Fatores Associados em Adultos de uma Cidade do Sul do Brasil: Estudo de Base Populacional.

Comportamentais - tabagismo: não-fumante, ex-fumante ou fumante (uso de um ou mais cigarros por dia no último mês); ingestão de álcool: abstêmio, uso moderado ou uso de risco (uso de uma ou mais doses padronizadas/ dia, para mulheres e duas ou mais doses padronizadas/ dia, para homens); atividade física: sedentário ou ativo ( $>150$ minutos de atividade física leve ou moderada ou 75 minutos de atividade física forte na última semana, obtido através do IPAQ curto (Hallal, Victora, Wells, \& Lima, 2003); crença religiosa: muito, moderadamente, pouco ou nada religioso (autopercepção); ter religião: sim ou não; freqüência religiosa: 0, 1-2, 3-4 e 5 ou mais vezes que freqüentou missão/culto/sessão religiosa nos últimos 30 dias.

Os dados foram duplamente digitados no programa EpiInfo 6.04, com checagem automática de consistência e amplitude. Para controle de qualidade, $10 \%$ das entrevistas foram repetidas pelos pesquisadores com uma versão resumida do questionário.

A análise estatística foi realizada através do pacote estatístico Stata. Após análise descritiva da amostra, foi feita análise bruta de associação do desfecho com as variáveis independentes, utilizando o teste de Wald para heterogeneidade e de tendência linear, no caso de variáveis categóricas ordinais. Para cada prevalência do desfecho foi calculado o intervalo de confiança de $95 \%$ (IC95\%), levando em consideração o efeito de delineamento.

O estudo foi aprovado pelo Comitê de Ética em Pesquisa da Faculdade de Medicina da Universidade Federal de Pelotas, tendo sido garantido sigilo das informa- ções e obtido consentimento por escrito de todos os participantes previamente à entrevista.

\section{Resultados}

Foram entrevistados 2.946 adultos, porém, quatro não forneceram informações sobre autopercepção de felicidade, resultando numa amostra final de 2.942 indivíduos.

A Tabela 1 descreve a amostra total de acordo com as variáveis independentes investigadas e segundo o sexo. Houve um predomínio da população feminina $(56,8 \%)$. Para ambos os sexos, a população estudada foi composta majoritariamente por indivíduos com cor da pele branca (cerca de 3/4 da população) e pelas categorias B e C da classificação econômica da ABEP, que representaram $80 \%$ da amostra, aproximadamente. O grupo com faixa etária maior de 60 anos de idade foi ligeiramente maior entre as mulheres, assim como o de indivíduos com nenhum ano de estudo, em comparação aos homens. Em relação à situação de trabalho, o desemprego foi discretamente mais comum entre as mulheres, ao passo que, diferentemente das mulheres, somente dois homens se definiram na categoria de "donas de casa". A proporção de mulheres que nunca fumou e que não consumiu álcool nos últimos 30 dias superou a de homens sensivelmente. Mais homens viviam na condição de casados/com companheira, enquanto o número de viúvas foi cerca de cinco vezes maior entre as mulheres. De maneira geral, as mulheres afirmavam ter religião, se consideravam mais religiosas e freqüentavam mais cultos/missas/sessões religiosas.

Tabela 1

Análise Descritiva da Amostra Estudada e Prevalência de Autopercepção de Felicidade em Adultos. Pelotas-RS, Brasil

\begin{tabular}{|c|c|c|c|c|c|c|}
\hline \multirow{3}{*}{ Variável } & \multicolumn{3}{|r|}{ Homens } & \multicolumn{3}{|c|}{ Mulheres } \\
\hline & \multirow[b]{2}{*}{$N$} & \multicolumn{2}{|c|}{ Autopercepção de felicidade } & \multicolumn{3}{|c|}{ Autopercepção de felicidade } \\
\hline & & $\%$ & $\%($ IC $95 \%)$ & $N$ & $\%$ & $\%(\mathrm{IC} 95 \%)$ \\
\hline Idade (anos) & 1270 & & & 1672 & & \\
\hline $20-29$ & 326 & 25,7 & $82,8(78,8-86,8)$ & 385 & 23,0 & $73,2(68,3-78,2)$ \\
\hline $30-39$ & 243 & 19,1 & $79,4(74,2-84,7)$ & 306 & 18,3 & $75,8(70,9-78,2)$ \\
\hline $40-49$ & 260 & 20,5 & $74,6(69,3-79,9)$ & 331 & 19,8 & $70,1(65,4-74,8)$ \\
\hline $50-59$ & 222 & 17,5 & $71,2(64,8-77,6)$ & 292 & 17,5 & $68,2(62,6-73,7)$ \\
\hline 60 ou mais & 219 & 17,3 & $66,7(60,2-73,1)$ & 358 & 21,4 & $70,7(65,5-75,8)$ \\
\hline Cor da pele & 1269 & & & 1669 & & \\
\hline Branca & 948 & 74,7 & $74,8(71,7-77,9)$ & 1271 & 76,2 & $72,3(69,5-75,1)$ \\
\hline Preta/Parda & 276 & 21,8 & $80,4(76,0-84,9)$ & 331 & 19,8 & $70,1(64,2-76,0)$ \\
\hline Outra & 45 & 3,6 & $64,4(50,8-78,0)$ & 67 & 4,0 & $65,7(52,2-79,0)$ \\
\hline Nível econômico (ABEP, 2005) & 1256 & & & 1657 & & \\
\hline A & 72 & 5,7 & $79,1(69,0-89,4)$ & 89 & 5,4 & $79,8(70,9-88,7)$ \\
\hline B & 414 & 33,0 & $78,0(73,4-82,7)$ & 512 & 30,9 & $75,8(71,7-79,9)$ \\
\hline $\mathrm{C}$ & 577 & 45,9 & $76,4(72,8-80,1)$ & 779 & 47,0 & $70,7(66,7-74,7)$ \\
\hline $\mathrm{D} / \mathrm{E}$ & 193 & 15,4 & $66,8(60,8-72,9)$ & 277 & 16,7 & $63,5(57,6-69,5)$ \\
\hline
\end{tabular}


(...Continuação)

\begin{tabular}{|c|c|c|c|c|c|c|}
\hline Escolaridade (anos) & 1270 & & & 1672 & & \\
\hline Zero & 52 & 4,1 & $55,7(43,4-68,1)$ & 121 & 7,2 & $57,0(48,0-66,0)$ \\
\hline $1-4$ & 220 & 17,3 & $70,0(63,4-76,6)$ & 289 & 17,3 & $73,4(67,8-78,9)$ \\
\hline $5-8$ & 427 & 33,6 & $75,9(71,7-80,0)$ & 500 & 29,9 & $67,6(63,2-72,0)$ \\
\hline $9-11$ & 329 & 25,9 & $79,6(74,9-84,4)$ & 431 & 25,8 & $74,0(69,1-78,9)$ \\
\hline 12 ou mais & 242 & 19,1 & $79,3(73,8-84,9)$ & 331 & 19,8 & $78,5(73,5-83,6)$ \\
\hline Situação de trabalho & 1270 & & & 1672 & & \\
\hline Desempregado & 98 & 7,7 & $67,3(58,4-76,3)$ & 170 & 10,2 & $61,2(53,1-69,2)$ \\
\hline Trabalhando & 929 & 73,2 & $78,1(75,4-80,9)$ & 823 & 49,2 & $75,7(72,3-79,1)$ \\
\hline Aposentado/pensionista ${ }^{a}$ & 178 & 14,0 & $65,7(58,5-73,0)$ & 262 & 15,7 & $66,0(59,3-72,7)$ \\
\hline Dona de casa ${ }^{b}$ & & & & 322 & 19,3 & $71,1(66,8-75,4)$ \\
\hline Outros & 65 & 5,1 & $80,0(70,4-89,6)$ & 95 & 5,7 & $72,6(62,7-82,6)$ \\
\hline Tabagismo & 1270 & & & 1672 & & \\
\hline Nunca fumou & 580 & 45,7 & $78,7(75,2-82,4)$ & 960 & 57,4 & $74,6(71,4-77,7)$ \\
\hline Fumante & 365 & 28,7 & $70,4(65,6-75,2)$ & 394 & 23,6 & $64,7(59,8-69,6)$ \\
\hline Ex-fumante & 325 & 25,6 & $76,0(71,7-80,3)$ & 318 & 19,0 & $71,4(66,5-76,3)$ \\
\hline Consumo de álcool & 1266 & & & 1663 & & \\
\hline Abstêmio & 453 & 35,8 & $73,3(68,8-77,8)$ & 1023 & 61,5 & $70,3(67,0-73,6)$ \\
\hline Consumo moderado & 724 & 57,2 & $77,3(74,3-80,4)$ & 587 & 35,3 & $74,6(70,9-78,3)$ \\
\hline Consumo de risco & 89 & 7,0 & $76,4(67,5-85,3)$ & 53 & 3,2 & $66,0(53,3-78,7)$ \\
\hline Sedentarismo (150 min) & 1264 & & & 1664 & & \\
\hline Não & 645 & 51,0 & $77,2(74,1-80,4)$ & 773 & 46,4 & $71,9(68,3-75,5)$ \\
\hline Sim & 619 & 49,0 & $74,3(70,4-78,2)$ & 891 & 53,6 & $71,5(68,5-74,5)$ \\
\hline Situação conjugal & 1261 & & & 1671 & & \\
\hline Casado/com companheiro & 871 & 68,6 & $75,9(72,7-79,1)$ & 928 & 55,5 & $75,0(72,1-77,9)$ \\
\hline Solteiro/sem companheiro & 297 & 23,4 & $77,4(73,2-81,7)$ & 367 & 22,0 & $71,7(66,4-77,0)$ \\
\hline Separado & 72 & 5,7 & $69,4(59,4-79,5)$ & 170 & 10,2 & $60,6(52,8-68,4)$ \\
\hline Viúvo & 29 & 2,3 & $65,5(48,2-82,8)$ & 206 & 12,3 & $66,0(59,1-72,9)$ \\
\hline Religião & 1269 & & & 1672 & & \\
\hline Não & 343 & 27,0 & $72,9(67,9-77,8)$ & 296 & 17,7 & $67,2(61,8-72,7)$ \\
\hline Sim & 926 & 73,0 & $76,7(73,7-79,7)$ & 1376 & 82,3 & $72,6(70,0-75,3)$ \\
\hline Crença religiosa & 1268 & & & 1669 & & \\
\hline Muito religioso & 205 & 16,2 & $75,6(69,8-81,4)$ & 475 & 28,5 & $76,0(71,5-80,5)$ \\
\hline Mais ou menos religioso & 509 & 40,1 & $77,8(74,3-81,3)$ & 727 & 43,6 & $71,3(67,9-74,6)$ \\
\hline Pouco religioso & 381 & 30,0 & $73,0(68,1-77,8)$ & 342 & 20,5 & $68,4(64,0-72,9)$ \\
\hline Nada religioso & 173 & 13,6 & $75,1(68,3-82,0)$ & 125 & 7,5 & $65,6(56,9-74,2)$ \\
\hline \multicolumn{7}{|l|}{ Freqüência religiosa nos últimos } \\
\hline 30 dias (número de vezes) & 1270 & & & 1669 & & \\
\hline Zero & 848 & 66,8 & $76,1(72,9-79,2)$ & 843 & 50,5 & $69,8(66,5-73,0)$ \\
\hline $1-2$ & 216 & 17,0 & $72,2(66,7-77,8)$ & 326 & 19,5 & $68,7(63,6-73,9)$ \\
\hline $3-4$ & 115 & 9,1 & $74,8(66,7-82,8)$ & 304 & 18,2 & $73,3(68,7-78,1)$ \\
\hline 5 ou mais & 91 & 7,2 & $81,3(73,0-89,9)$ & 196 & 11,7 & $82,1(77,2-87,1)$ \\
\hline \multicolumn{7}{|l|}{ Número de habitantes } \\
\hline no domicílio & 1264 & & & 1666 & & \\
\hline Um & 77 & 6,1 & $68,8(58,7-78,9)$ & 155 & 9,3 & $71,0(63,6-78,3)$ \\
\hline Dois & 249 & 19,7 & $76,7(71,6-81,8)$ & 350 & 21,0 & $68,9(63,3-74,4)$ \\
\hline Três & 349 & 27,6 & $74,8(69,6-80,0)$ & 447 & 26,8 & $74,3(70,0-78,5)$ \\
\hline Quatro & 297 & 23,5 & $77,1(71,7-82,6)$ & 367 & 22,0 & $74,4(69,7-79,0)$ \\
\hline Cinco ou mais & 292 & 23,1 & $76,0(70,9-81,2)$ & 347 & 20,8 & $68,6(63,2-74,0)$ \\
\hline
\end{tabular}

${ }^{a}$ Entre os homens não haviam pensionistas. "Dois homens referiram ser "Dona de casa", tendo sido categorizados como "Outros". 
Scalco, D. L., Araújo, C. L. \& Bastos, J. L. (2011). Autopercepção de Felicidade e Fatores Associados em Adultos de uma Cidade do Sul do Brasil: Estudo de Base Populacional.

A distribuição quanto às faces apontadas, indicando níveis de autopercepção de felicidade, está apresentada na Figura 2. A prevalência global de indivíduos felizes foi de 73,4\% (IC95\% 71,2; 75,5), sendo 71,6\% (IC95\% $69,0 ; 74,1)$ entre as mulheres e 75,7\% (IC95\% 73,0; 78,2) entre os homens.
A Tabela 1 também apresenta as prevalências de felicidade para cada categoria das variáveis independentes, com seus respectivos IC95\%. A Tabela 2 mostra as razões de prevalência e respectivos valores- $p$.

\section{Autopercepção de felicidade segundo uma escala de faces em uma população de adultos. Pelotas-RS. Brasil, 2008.}

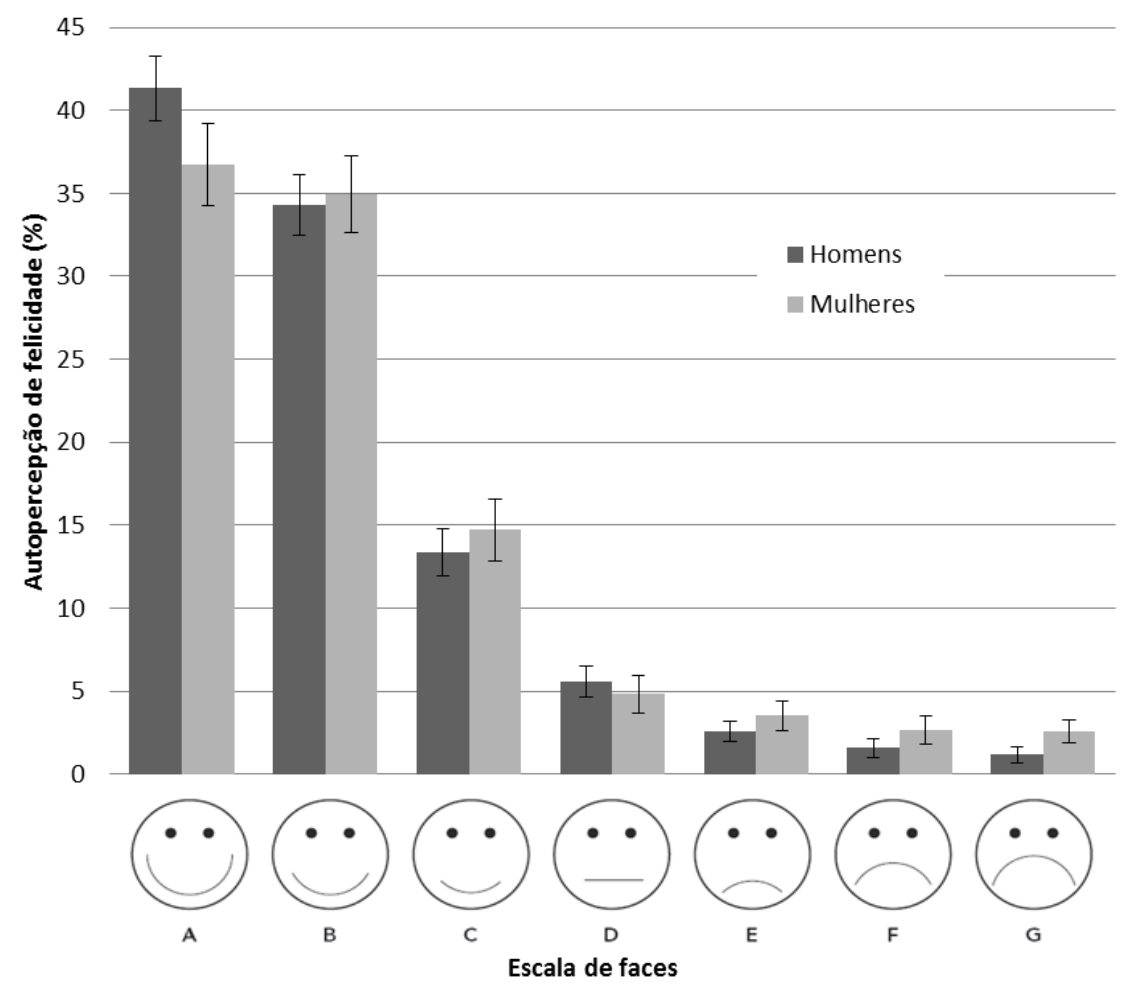

Figura 2. Autopercepção de felicidade segundo uma Escala de Faces em uma População de Adultos. Pelotas-RS, Brasil, 2008.

Tabela 2

Análise Bruta das Razões de Prevalência de Autopercepção de Felicidade e Variáveis Independentes em Adultos. Pelotas-RS, Brasil

\begin{tabular}{|c|c|c|c|c|}
\hline \multirow[t]{2}{*}{ Variável } & \multicolumn{2}{|c|}{ Homens } & \multicolumn{2}{|c|}{ Mulheres } \\
\hline & $\mathrm{RP}^{*}(\mathrm{IC} 95 \%)$ & Valor $p$ & RP (IC 95\%) & Valor $p$ \\
\hline Idade (anos) & & $0,001^{\mathrm{a}}$ & & $0,165^{\mathrm{a}}$ \\
\hline $20-29$ & Referência & & Referência & \\
\hline $30-39$ & $0,96(0,88-1,04)$ & & $1,04(0,95-1,13)$ & \\
\hline $40-49$ & $0,90(0,83-0,98)$ & & $0,96(0,87-1,05)$ & \\
\hline $50-59$ & $0,86(0,78-0,95)$ & & $0,93(0,84-1,04)$ & \\
\hline 60 ou mais & $0,80(0,73-0,89)$ & & $0,96(0,88-1,06)$ & \\
\hline Cor da pele & & $0,019^{b}$ & & $0,463^{\mathrm{b}}$ \\
\hline Branca & Referência & & Referência & \\
\hline Preta/Parda & $1,08(1,00-1,15)$ & & $0,97(0,88-1,06)$ & \\
\hline Outra & $0,86(0,69-1,07)$ & & $0,91(0,74-1,11)$ & \\
\hline
\end{tabular}


(...Continuação)

Nível econômico (ABEP, 2005)

A

B

C

$\mathrm{D} / \mathrm{E}$

Escolaridade (anos)

Zero

$1-4$

5-8

9-11

12 ou mais

Situação de trabalho

Trabalhando

Desempregado

Aposentado/pensionista ${ }^{c}$

Dona de casa ${ }^{\mathrm{d}}$

Outros

Tabagismo

Nunca fumou

Fumante

Ex-fumante

Consumo de álcool

Abstêmio

Consumo moderado

Consumo de risco

Sedentarismo

Não

Sim

Situação conjugal

Casado/com companheiro

Solteiro/sem companheiro

Separado

Viúvo

Religião

Não

Sim

Crença religiosa

Nada religioso

Pouco religioso

Mais ou menos religioso

Muito religioso

Freqüência religiosa nos últimos

30 dias (número de vezes)

Zero

$1-2$

$3-4$

5 ou mais

Número de habitantes no domicílio

Um

Dois

Três

Quatro

Cinco ou mais
$1,18(1,01-1,38)$

$1,17(1,05-1,30)$

$1,14(1,03-1,27)$

Referência

Referência

$1,26(0,99-1,58)$

$1,36(1,10-1,69)$

$1,43(1,13-1,80)$

$1,42(1,21-1,79)$

Referência

$0,86(0,75-0,99)$

$0,84(0,75-0,94)$

$1,02(0,91-1,15)$

Referência

$0,89(0,82-0,97)$

$0,96(0,90-1,03)$

Referência

$1,06(0,98-1,13)$

$1,04(0,92-1,18)$

Referência

$0,96(0,90-1,02)$

Referência

1,02 (0,96-1,09)

$0,92(0,79-1,07)$

$0,86(0,66-1,13)$

Referência

$1,05(0,97-1,14)$

Referência

$1,01(0,89-1,13)$

$1,04(0,93-1,15)$

$0,97(0,87-1,08)$

$0,199^{b}$

$0,315^{\text {b }}$

$0,229^{\mathrm{b}}$

$0,335^{\mathrm{b}}$

$0,397^{\mathrm{b}}$

$$
0,350^{\text {b }}
$$

Referência

0,95 (0,87-1,03)

$0,98(0,88-1,10)$

$1,07(0,95-1,20)$

$0,91(0,77-1,07)$

$1,01(0,92-1,11)$

$0,98(0,89-1,08)$

1,01 (0,92-1,12)

Referência

$0,732^{\mathrm{b}}$ $0,001^{\mathrm{a}}$

$1,26(1,08-1,46)$

$1,19(1,07-1,33)$

1,11 (0,99-1,25)

Referência

Referência

1,29 (1,08-1,53)

$1,19(1,01-1,40)$

$1,30(1,10-1,54)$

$1,38(1,17-1,62)$

Referência

$0,80(0,70-0,93)$

$0,87(0,78-0,97)$

$0,94(0,87-1,01)$

$0,96(0,84-1,10)$

Referência

$0,87(0,80-0,94)$

$0,96(0,89-1,03)$

Referência

$1,06(0,99-1,13)$

$0,94(0,78-1,14)$

$0,001^{\mathrm{a}}$

$0,003^{\mathrm{b}}$

$0,144^{\mathrm{b}}$

Referência

0,99 (0,94-1,05)

Referência

$0,96(0,88-1,04)$

$0,81(0,71-0,92)$

$0,88(0,79-0,99)$

$0,839^{\mathrm{b}}$

$0,006^{\mathrm{b}}$

Referência

1,08 (1,00-1,17)

Referência

$1,04(0,90-1,20)$

$1,09(0,94-1,25)$

$1,16(1,01-1,33)$

$0,064^{\mathrm{b}}$

$0,005^{\mathrm{a}}$

Referência

$0,99(0,91-1,06)$

$1,05(0,97-1,13)$

$1,18(1,09-1,27)$

$1,03(0,92-1,17)$

$1,00(0,89-1,13)$

$1,08(0,99-1,18)$

1,08 (0,98-1,20)

Referência

Notas: * RP (razão de prevalência). ${ }^{a}$ Teste de Wald para tendência linear. ${ }^{b}$ Teste de Wald para heterogeneidade. ${ }^{c}$ Entre os homens não haviam pensionistas. 'Dois homens referiram ser "Dona de casa", tendo sido categorizados como "Outros". 
Conforme a Tabela 2, é possível observar que, tanto entre os homens como entre as mulheres, a prevalência de indivíduos felizes foi maior entre aqueles com maior escolaridade e maior nível socioeconômico, assim como foi menor entre desempregados, aposentados e fumantes. Maiores prevalências de felicidade estiveram associadas a cor da pele preta/parda e com indivíduos mais jovens, entre os homens. Diferentemente, mulheres separadas ou viúvas apresentaram menores prevalências de felicidade, enquanto que mulheres mais religiosas condição essa definida através da crença religiosa autopercebida e pela freqüência religiosa - apresentaram maiores prevalências de felicidade. Nível de atividade física, consumo de álcool e o número de habitantes de domicílio não se mostraram associados com o desfecho em ambos os sexos.

\section{Discussão}

A alta taxa de resposta observada neste estudo, bem como o caráter aleatório das perdas e recusas identificadas contribuem para a representatividade da amostra. Além disso, comparando-se a distribuição da amostra, por sexo e faixa etária, com dados da população residente em Pelotas, obtidos no Censo de 2000 do IBGE e em estudos transversais de base populacional realizados anteriormente (Duquia, Menezes, Reichert, \& Almeida, 2007), não foram encontradas diferenças importantes, o que reforça a validade interna do estudo.

Deve-se atentar para o fato de que este estudo visa avaliar as chamadas variáveis situacionais (teorias "bottomup") - variáveis independentes - que são responsáveis por apenas 10 a $15 \%$ da variância de felicidade ou de construtos correlatos (Argyle, 1999; Diener, 1984). Não foram abordados os processos psicológicos, que, segundo as teorias "top-down", seriam os responsáveis por uma maior modulação da felicidade, frente às influências situacionais que os indivíduos vivenciam (Diener, 1984).

Além desta, outras possíveis limitações ou críticas ao presente estudo devem ser apontadas. As associações observadas nas análises bivariáveis não devem ser interpretadas como relações de causa e efeito. Pressupor causalidade é complexo e envolve algumas considerações. A precedência temporal da causa em relação ao efeito e o entendimento de que uma série de fatores pode interagir de modo bastante intrincado na determinação do desfecho são dois exemplos. Desta forma, este estudo propõe-se tão somente a identificar grupos populacionais cuja prevalência de felicidade autopercebida é maior ou menor.

Um possível viés nas respostas poderia ser a contaminação pelo estado de ânimo ou outros fatores situacionais momentâneos. No entanto, outros estudos têm mostrado que é grande a estabilidade de autopercepção de felicidade e construtos correlatos ao longo do tempo (KoivumaaHonkanen, Kaprio, Honkanen, Viinamäki, \& Koskenvuo, 2005). Eid e Diener (1999) encontraram que fatores situacionais têm pouca influência em comparação com fatores que influenciam medidas de bem estar subjetivo por longo tempo. A padronização da coleta dos dados e a inserção da questão sobre felicidade no início do questionário foram realizados de modo a minimizar possíveis interferências.

Os principais achados do presente estudo foram: a alta prevalência de autopercepção de felicidade na população estudada, a associação positiva entre ser feliz e ter maior escolaridade e ser de nível econômico mais elevado e, a associação negativa entre ser feliz e estar desempregado para ambos os sexos, o decréscimo da prevalência de felicidade com o aumento da idade entre os homens e a associação negativa entre ser separada ou viúva e autoperceber-se feliz entre as mulheres.

A prevalência encontrada de adultos felizes foi de $73 \%$ da população, resultado esse que corrobora achados de outros estudos que indicam que a grande maioria dos indivíduos consideram-se felizes. Cummins (1998) fez uma revisão com busca sistemática de mais de 2000 artigos, selecionando 206 de vários países que apresentavam dados sobre satisfação de vida. A partir desses construiu um índice, que utilizou para chegar a um valor médio mundial de felicidade de $70 \%$ (erro padrão de $5 \%$ ). Se aplicado o mesmo índice ao presente estudo, seu valor seria de $81,0 \%$ (IC95\% 80,2; 81,8\%), mais alto do que a média global. Como já enfatizado, tais comparações devem ser cautelosas, o que não reduz a plausibilidade de que a grande maioria das pessoas se autopercebe feliz.

A tendência de maior prevalência de pessoas felizes entre os homens mais jovens foi marcante. No entanto, isto não se verificou entre as mulheres. Outros estudos não demonstraram um padrão consistente de variação de felicidade com a idade. Em um estudo transversal realizado na China, sem estratatificação por sexo, Lu (1995) encontrou que a satisfação de vida foi maior entre os idosos. Yang (2008), a partir da análise de uma série de inquéritos consecutivos sobre felicidade, nos Estados Unidos da América, também mostrou um incremento dos níveis de felicidade com o aumento da idade, após controle para efeitos de coorte e de período, tanto entre homens como entre mulheres.

A associação entre felicidade e nível econômico, assim como com escolaridade, também foi significativa. Resultados similares ao presente estudo, foi encontrado entre adolescentes de Pelotas (Silva et al., 2007): utilizando instrumento semelhante, a pesquisa mostrou que indivíduos pertencentes a classes socias mais privilegiadas apresentaram maior prevalência de bem-estar psicológico. Outros estudos realizados em diferentes países obtiveram achados semelhantes, tanto para nível econômico como para escolaridade (Dear, Henderson, \& Korten, 2002; Lipovcan, Brkljacic, \& Sakic, 2007; Subramanian, Kim, \& Kawachi, 2005). Os autores do presente estudo compartilham a hipótese levantada por Myers (2000) de que tal associação seja especialmente importante em países com maior desigualdade social, nos quais as cama- 
das mais pobres da população não têm acesso a necessidades básicas como, por exemplo, a alimentação. Destaca-se também, nesta pesquisa, os níveis comparativamente reduzidos de indivíduos felizes entre o grupo com nenhum ano de escolaridade.

Assim como encontrado em outro estudo (Dear et al., 2002), o desemprego esteve associado com menores níveis de autopercepção de felicidade. Lucas, Clark, Georgellis e Diener (2004), através de uma análise prospectiva da satisfação de vida informada por mais de 20.000 pessoas, na Alemanha, durante um período de 15 anos, observou que o desemprego foi capaz de reduzir os níveis de satisfação de vida por longos períodos. Ser aposentado/pensionista também mostrou comportamento semelhante em relação à autopercepção de felicidade. Utilizando o mesmo banco de dados do estudo de Lucas et al. (2004), Pinquart e Schindler (2007) concluíram que, embora a maioria dos adultos experienciaram a aposentadoria com discreto aumento inicial nos níveis de satisfação de vida, o comportamento para cada indivíduo foi muito dependente da interação com as circunstâncias externas e fontes de recursos individuais como situação conjugal e estado de saúde.

A situação conjugal, em relação às mulheres, mostrou forte associação com a felicidade autopercebida. Outros estudos (Ballas \& Dorling, 2007) já têm mostrado que divórcio ou separação, assim como a perda do companheiro(a), são considerados eventos de vida estressantes, que impactam negativamente na avaliação positiva que os indivíduos fazem de suas vidas. Curioso observar que tais diferenças não ocorreram entre os homens. Entretanto, a ausência de tal associação, ao menos entre o grupo de viúvos e casados/com companheiro, pode ter ocorrido devido a baixa prevalência de viúvos.

Entre as mulheres, a crença religiosa e maior freqüência a cultos religiosos estiveram associadas positivamente com felicidade, ainda que com razões de prevalência não muito altas. Uma ampla revisão sistemática, conduzida por Moreira-Almeida, Neto e Koenig (2006), aponta que maiores níveis de envolvimento religioso estão associados positivamente a indicadores de bem-estar psicológico.

Outras associações com autopercepção de felicidade foram detectadas no presente estudo. A ausência de destaque pelos autores é intencional, pois, ainda que existam, refletem razões de prevalência relativamente baixas. Entre estas, citam-se as associações positivas de maiores prevalências de autopercepção de felicidade com ser do sexo masculino, classificar-se nas categorias de cor da pele preta ou parda para os homens e, nunca ter fumado, para ambos os sexos. Esta última é a única destas associações reproduzida de forma mais consistente em outros estudos (Dear et al., 2002).

Partindo-se do pressuposto de que estar feliz é uma condição associada a desfechos positivos em saúde, além de ser um fim por si só, torna-se importante identificar quem são as pessoas felizes e suas principais características. Tal conhecimento poderia ajudar na elaboração de políticas e estratégias que visem aumentar o bem-estar populacional por permitir a visualização de grupos populacionais mais vulneráveis em termos de saúde. Além disso, os resultados deste estudo podem contribuir para a formulação de hipóteses sobre os determinantes de felicidade, abrindo caminho para a realização de estudos epidemiológicos com desenhos prospectivos, com maior capacidade de identificar adequadamente associações causais.

\section{Referências}

Argyle, M. (1999). Causes and correlates of happiness. In D. Kahneman, E. Diener, \& N. Schwars (Eds.), Well-being: The foundations of hedonic psychology (pp. 354-373). New York: Russel Sage Foundation.

Associação Brasileira de Empresas de Pesquisa. (2005). Critério Brasileiro de Classificação Econômica. Retrieved October 26, 2008, from http://www.abep.org

Ballas, D., \& Dorling, D. (2007). Measuring the impact of major life events upon happiness. International Journal of Epidemiology, 36(6), 1244-1252.

Barak, Y. (2006). The immune system and happiness. Autoimmunity Reviews, 5(8), 523-527.

Barros, A. J. D., Menezes, A. M. B., Santos, I. S., Assunção, M. C. F., Gigante, D., Fassa, A. G., et al. (2008). O Mestrado do Programa de Pós-graduação em Epidemiologia da UFPel baseado em consórcio de pesquisa: Uma experiência inovadora. Revista Brasileira de Epidemiologia, 11(1), 133-144.

Cowley, G. (2002, September 18). The science of happiness. Newsweek, 140(12), 46-48.

Cummins, R. A. (1998). The second approximation to an international standard for life satisfaction. Social Indicators Research, 43(3), 307-334.

Danner, D. D., Snowdon, D. A., \& Friesen, W. V. (2001). Positive emotions in early life and longevity: Findings from the nun study. Journal of Personality and Social Psychology, 80(5), 804-813.

Dear, K., Henderson, S., \& Korten, A. (2002). Well-being in Australia: Findings from the National Survey of Mental Health and Well-being. Social Psychiatry and Psychiatric Epidemiology, 37(11), 503-509.

Delamothe, T. (1996). Happiness. British Medical Journal, 331(7531), 1489-1490.

Diener, E. (1984). Subjective well-being. Psychological Bulletin, 95(3), 542-575.

Diener, E. (2000). Subjective well-being. The science of happiness and a proposal for a national index. American Psychologist, 55(1), 34-43.

Duquia, R. P., Menezes, A. M. B., Reichert, F. F., \& Almeida, H. L., Jr. (2007). Prevalence and associated factors with sunscreen use in Southern Brazil: A population-based study. Journal of the American Academy of Dermatology, 57(1), 73-80.

Eid, M., \& Diener, E. (1999). Intraindividual variability in affect: Reliability, validity, and personality correlates. Journal of Personality and Social Psychology, 76(4), 662-676.

Fresneda, C. (2007, 10 febrero). El mundo que viene. Opinión. El Mundo, p. 8. 
Scalco, D. L., Araújo, C. L. \& Bastos, J. L. (2011). Autopercepção de Felicidade e Fatores Associados em Adultos de uma Cidade do Sul do Brasil: Estudo de Base Populacional.

Geraldo, P. H. B. (s.d.). O utilitarismo e suas criticas: Uma breve revisão. Retrieved October 26, 2008, from http:// www.conpedi.org/manaus/arquivos/Anais/Pedro\%20Heitor $\% 20$ Barros $\% 20$ Geraldo_Teoria\%20da $\% 20 J u s t i c a . p d f$

Hallal, P. C., Victora, C. G., Wells, J. C., \& Lima, R. C. (2003). Physical inactivity: Prevalence and associated variables in Brazilian adults. Medicine \& Science in Sports \& Exercise, 35(11), 1894-1900.

Instituto Brasileiro de Geografia e Estatística. (2000). Censo 2000. Retrieved October 26, 2008, from http://www.ibge.gov.br Instituto Brasileiro de Geografia e Estatística. (2007). IBGE Cidades@: Pelotas, RS. Retrieved from http://www.ibge. gov.br/cidadesat/painel/painel.php?codmun=431440\#

Koivumaa-Honkanen, H., Honkanen, R., Viinamaki H., Heikkila, K., Kaprio, J., \& Koskenvuo, M. (2000). Selfreported life satisfaction and 20-year mortality in healthy Finnish adults. American Journal of Epidemiology, 152(10), 983-991.

Koivumaa-Honkanen, H., Kaprio, J., Honkanen, R. J., Viinamäki, H., \& Koskenvuo, M. (2005). The stability of life satisfaction in a 15 -year follow-up of adult healthy at baseline. BMC Psychiatry, 5, 4.

Lipovcan, L. K., Brkljacic, T., \& Sakic, V. (2007). Monthly income and subjective well-being of Croatian citizens. Croatian Medical Journal, 48(5), 727-733.

Lu, L. (1995). The relationship between subjective well-being and psychosocial variables in Taiwan. Journal of Social Psychology, 135(3), 351-357.

Lucas, R. E., Clark, A. E., Georgellis, Y., \& Diener, E. (2004). Unemployment alters the set point for life satisfaction. Psychological Science, 15(1), 8-13.

Lucas, R. E., Diener, E., \& Suh, E. (1996). Discriminant validity of well-being measures. Journal of Personality and Social Psychology, 71(3), 616-628.

Mancall, M. (2004). Gross National Happiness and Development: An essay. In K. Ura \& K Galay (Eds.), Gross national happiness and development (pp. 1-50). Thimphu, Bhutan: The Centre for Bhutan Studies.

McDowell, I., \& Newell, C. (1996). Measuring health: A guide to rating scales and questionnaires. New York: Oxford University Press.

McKevitt, C., Redfern, J., La-Placa, V., \& Wolfe, C. D. A. (2003). Defining and using quality of life: A survey of health care professionals. Clinical Rehabilitation, 17(8), 865-870.

Moreira, C. A. (2007, 22 abr.). Felicidade: Sempre falta um pedacinho. Donna ZH. Zero Hora, p. 8.

Moreira-Almeida, A., Neto, F. L., \& Koenig, H. G. (2006). Religiousness and mental health: A review. Revista Brasileira de Psiquiatria, 28(3), 242-250.

Myers, D. G. (2000). The funds, friends, and faith of happy people. American Psychologist, 55(1), 56-67.

Nettle, D. (2005). Happiness: The science behind your smile. Oxford, UK: Oxford University Press.

Pinquart, M., \& Schindler, I. (2007). Changes of life satisfaction in the transition to retirement: A latent-class approach. Psychology and Aging, 22(3), 442-455.

Sandvik, E., Diener, E., \& Seidlitz, L. (1993). Subjective wellbeing: The convergence and stability of self-report and nonself-report measures. Journal of Personality, 61(3), 317-342.

Seymour, L. (2007). Health, wealth and the pursuit of happiness. Journal of the Royal Society for the Promotion of Health, $127(2), 61-62$.
Silva, R. A., Horta, B. L., Pontes, L. M., Faria, A. D., Souza, L. D. M., Cruzeiro, A. L. S., et al. (2007). Bem-estar psicológico e adolescência: Fatores associados. Cadernos de Saúde Pública, 23(5), 1113-1118.

Subramanian, S. V., Kim, D., \& Kawachi, I. (2005). Covariation in the socioeconomic determinants of self rated health and happiness: A multivariate multilevel analysis of individuals and communities in the USA. Journal of Epidemiology \& Community Health, 59(8), 664-669.

Veenhoven, R. (1997). Progrès dans la comprehénsion du bonheur. Revue Québecoise de Psychologie, 18, 29-74.

Yang, Y. (2008). Social inequalities in happiness in the United States, 1972 to 2004: An age-period-cohort analysis. American Sociological Review, 73, 203-226. 